\title{
Neurensin-2 promotes proliferation, invasion and migration of colorectal cancer cells via interaction with SOX12
}

\author{
GANG WANG and KAI YANG
}

\author{
Department of General Surgery, The First Affiliated Hospital of \\ Jinzhou Medical University, Jinzhou, Liaoning 121001, P.R. China
}

Received June 12, 2020; Accepted September 24, 2020

DOI: $10.3892 / \mathrm{ol} .2020 .12252$

\begin{abstract}
Colorectal cancer (CRC) is the third most common malignant type of tumor worldwide. Neurensin-2 (NRSN2) is a small neuronal membrane protein associated with tumorigenesis. Therefore, the present study aimed to investigate the association between NRSN2 and CRC, and further examined the underlying mechanism of its effect on CRC metastasis. Human CRC SW620 cells were used to determine the biological functions of NRSN2 in CRC. Cell counting Kit-8 (CCK8), colony formation, wound-healing and transwell assays were performed to evaluate the role of NRSN2 on survival and metastasis of SW620 cells. The interaction between NRSN2 and SOX12 was determined via bioinformatics analysis and confirmed using immunoprecipitation. It was identified that NRSN2 was highly expressed in CRC cells and served a critical role in CRC cell survival compared with in healthy colon epithelial cells. Furthermore, NRSN2-knockdown inhibited the proliferation, invasion and migration of SW620 cells, while NRSN2 overexpression promoted these cellular processes. Additionally, it was demonstrated that NRSN2 could recruit SOX12 in SW620 cells. NRSN2-knockdown decreased SOX12 expression, while NRSN2 overexpression upregulated SOX12 expression. Overall, the present results suggested NRSN2 as a novel biomarker for CRC diagnosis and identified NRSN2 as a potential therapeutic target for CRC treatment.
\end{abstract}

\section{Introduction}

Colorectal cancer (CRC) was the third most common malignant type of tumor worldwide in 2017 (1-3), and mainly results from metastasis (4,5). For advanced CRC, 5-year survival rates have been reported to be $<50 \%$ in 2019 (6). Accumulating evidence has suggested that aging and rapid population growth contribute to high morbidity and mortality rates

Correspondence to: Dr Gang Wang, Department of General Surgery, The First Affiliated Hospital of Jinzhou Medical University, 2 Section 5 Renmin Street, Jinzhou, Liaoning 121001, P.R. China E-mail: wangzhiyong2004@yeah.net

Key words: neurensin-2, SOX12, colorectal cancer both in high-income countries and, especially, in low- and middle-income countries $(7,8)$. According to a previous report, $\sim 1.5$ million individuals are diagnosed with CRC each year (9). Furthermore, the 5-year survival rate is $<44 \%$ after resection of hepatic colorectal metastases $(10,11)$. Although progress has been achieved in understanding the potential biochemical mechanism of CRC $(12,13)$, current clinical diagnosis and gene-targeted or pharmacologic therapies present numerous limitations in decreasing the mortality rate of patients with CRC. Therefore, it is urgent to develop novel and effective therapeutic strategies for CRC.

Neurensin-2 (NRSN2) is a small neuronal membrane protein located in small vesicles of neural cells (14). A previous study has revealed that NRSN2 is highly expressed in osteosarcoma tissues and promotes osteosarcoma cell proliferation via dysregulation of the PI3K/Akt/mTOR and $\mathrm{Wnt} / \beta$-catenin signaling pathways (14). Furthermore, Tang et al (15) reported that NRSN2 is closely associated with the malignant phenotype of ovarian cancer. However, contradictory roles of NRSN2 have been observed in non-small cell lung cancer (NSCLC) and hepatocellular carcinoma (HCC). For example, a previous study suggested that NRSN2 is upregulated in malignant tissues and promotes NSCLC cell proliferation via the PI3K/Akt/mTOR pathway (16). By contrast, Wang et al (17) demonstrated that NRSN2 is downregulated in HCC tissues and exerts a suppressive role in HCC tumorigenesis. These findings indicate that NRSN2 may serve a pivotal role in tumorigenesis, but its role in CRC is yet to be elucidated.

To further investigate the potential value of NRSN2 in CRC, the present study selected the CRC SW620 cell line. The aim of present study was to investigate the association between NRSN2 and CRC and further investigate the underlying mechanism of its effect on CRC metastasis.

\section{Materials and methods}

Cell culture and transfection. The human CRC SW480, SW620 and HCT116 cell lines, and the human normal colon epithelial FHC cell line were obtained from the American Type Culture Collection (ATCC). Cells were cultured in DMEM containing 10\% FBS (both HyClone; Cytiva), $100 \mu \mathrm{g} / \mathrm{ml}$ streptomycin and $100 \mathrm{U} / \mathrm{ml}$ penicillin with a $5 \% \mathrm{CO}_{2}$ atmosphere at $37^{\circ} \mathrm{C}$.

SW620 cells were transfected in 6-well plates with short hairpin (sh) RNA-NRSN2 (100 pmol), shRNA-NC (100 pmol) 
or overexpression-NRSN2 (Ov-NRSN2; $100 \mathrm{pmol})$ and Ov-NC (100 pmol) for modulating NRSN2 expression. shRNA-NRSN2 causes downregulation of NRSN2 expression, while Ov-NRSN2 is a plasmid that specifically elevates NRSN2 expression. The sequences are as follows: shRNA-NRSN2-1 (5'-GCACCTCTT CTATGAGGACTG-3'); shRNA-NRSN2-2 (5'-GCAATCTTC TGTGCAGACTAT-3'); shRNA-NC (scrambled sequence, 5'-TTCTCCGAACGTGTCACGT-3'). Ov-NRSN2 plasmid (pRK5-NRSN2, forward, 5'-CTAGCTAGCATGATGCCGAG CTGCAATC-3' and reverse, 5'-CGCGGATCCTCAGGAGTC CCTCTTGGG-3') and Ov-NC (empty vector, pRK5-control plasmid) were constructed by Shanghai GenePharma, Co., Ltd. After SW620 cells were grown to $\sim 80 \%$ confluence, transfection was performed using Lipofectamine ${ }^{\circledR} 2000$ (Invitrogen; Thermo Fisher Scientific, Inc.) at $37^{\circ} \mathrm{C}$ according to the manufacturer's protocol. At $24 \mathrm{~h}$ post-transfection, cells were harvested for subsequent experimentation and RT-qPCR was used to detect the efficiency of transfection.

Reverse transcription-quantitative PCR (RT-qPCR). Total RNA was extracted using TRIzol ${ }^{\circledR}$ reagent (Invitrogen; Thermo Fisher Scientific, Inc.), according to the manufacturer's protocol. RT was performed to synthesize cDNA with a Reverse Transcription kit (Thermo Fisher Scientific, Inc.), and qPCR was performed using SYBR Green Real-Time PCR Master Mix (Bio-Rad Laboratories, Inc.)and primers for NRSN2 and GAPDH (Thermo Fisher Scientific, Inc.) according to the manufacturer's instructions. The expression levels of NRSN2 were normalized to those of GAPDH from the same RT sample and were detected using an Applied Biosystems 7500 Real Time PCR system (Applied Biosystems; Thermo Fisher Scientific, Inc.) with a SYBR Green QPCR Master mix (Thermo Fisher Scientific, Inc.). The relative expression levels were calculated using the $2^{-\Delta \Delta \mathrm{Cq}}$ method (18). qPCR amplification parameters were: annealing at $25^{\circ} \mathrm{C}$ for $10 \mathrm{~min}$, extension at $42^{\circ} \mathrm{C}$ for $30 \mathrm{~min}$; inactivation at $85^{\circ} \mathrm{C}$ for $10 \mathrm{~min}$, after extension of the detection of fluorescence signals, a total of 40 cycles. Primer sequences were as follows: NRSN2 forward, 5'-GATGGCAAGTGGTATGGGGTC-3' and reverse, 5'-CGAGGACAGGCTGATCTTCC-3'; and GAPDH forward, 5'-CCAGGTGGTCTCCTCTGA-3' and reverse, 5'-GCTGTAG CCAAATCGTTGT-3'.

Western blot analysis. SW620 cells were harvested and lysed using RIPA lysis buffer containing $1 \mathrm{mM}$ PMSF, $1 \mu \mathrm{M}$ Ben, $1 \mathrm{mM} \mathrm{Na} \mathrm{VO}_{4}, 10 \mu \mathrm{g} / \mathrm{ml}$ leupetin and $10 \mu \mathrm{g} / \mathrm{ml}$ Aprotinin. Protein concentration was measured using a BCA protein assay (Thermo Fisher Scientific, Inc.). Total protein ( $25 \mu \mathrm{g} /$ lane) was separated via $12 \%$ SDS-PAGE and transferred to PVDF membranes. Membranes were blocked with 5\% skimmed milk at room temperature for $2 \mathrm{~h}$. Subsequently, appropriate primary antibody dilutions $(1: 1,000)$ were prepared and incubated with membranes overnight at $4^{\circ} \mathrm{C}$. The antibodies included rabbit anti-NRSN2 (1:1,000; cat. no. ab237739; Abcam), rabbit anti-MMP2 (1:1,000; cat. no. 4022; Cell Signaling Technology, Inc.), rabbit anti-MMP9 (1:1,000; cat. no. 3852; Cell Signaling Technology, Inc.), rabbit anti-SOX12 (1:1,000; cat. no. SAB4502835; Sigma-Aldrich; Merck KGaA) and rabbit anti-GAPDH (1:1,000; cat. no. 5014; Cell Signaling Technology, Inc.). After washing with PBS three times, the membranes were incubated with HRP-conjugated goat anti-rabbit IgG secondary antibodies (1:1,000; cat. no. 21537; EMD Millipore) for $2 \mathrm{~h}$ at room temperature. The membranes were visualized using Tanon-5200 Chemiluminescence Imager (Tanon Science and Technology Co., Ltd.) with ECL western blotting substrate (EMD Millipore). Band intensity was quantified using ImageJ v1.8.0 (National Institutes of Health) and relative protein levels were normalized to GAPDH.

Immunoprecipitation (IP). The target proteins of NRSN2 were predicted using the STRING database (https://string-db. org/cgi/network). For IP, cells lysed with lysis buffer (20 mM Tris- $\mathrm{HCl}$ (pH 8.0), $300 \mathrm{mM} \mathrm{NaCl}, 1$ mM EDTA and $0.5 \%$ Nonidet P-40). After centrifugation $(12,000 \times \mathrm{g}$ for $10 \mathrm{~min}$ at $4^{\circ} \mathrm{C}$ ), $2 \mathrm{mg}$ cell lysate supernatants were incubated with Anti-FLAGM2 Magnetic beads (cat. no. M8823, Sigma-Aldrich; Merck KGaA) or protein A/G Sepharose beads ( $20 \mu 1$, cat. no. sc-2003, Santa Cruz Biotechnology, Inc.) according to manufacturer's protocol and then with primary antibodies for $12 \mathrm{~h}$ at $4^{\circ} \mathrm{C}$, and the collected beads were washed four times with washing buffer $(0.05 \%$ Triton X-100 immunoprecipitation buffer without a protease inhibitor cocktail), using IgG as a blank control. Immunoprecipitates were boiled in sample loading buffer with 1X SDS loading buffer (50 $\mu \mathrm{l})$ for $5 \mathrm{~min}$. Captured proteins on Sepharose beads were then separated via 8\% SDS-PAGE and western blot analysis was performed as previously mentioned.

Cell viability. A Cell Counting Kit-8 assay (CCK-8; Thermo Fisher Scientific, Inc.) was performed to detect the cell viability. In brief, SW620 cells were seeded into a 96-well plate at a density of 5,000 cells/well. All experiments were performed $\geq 3$ times in triplicate. After transfection, cell viability at 24,48 and $72 \mathrm{~h}$ was determined using a CCK-8 assay. CCK-8 reagent $(10 \mu \mathrm{l})$ was added per well and incubated with the cells for another $2 \mathrm{~h}$ according to the manufacturer's protocol.

Colony formation assay. SW620 cells $\left(5 \times 10^{3}\right.$ cells $\left./ \mathrm{ml}\right)$ were seeded in 6-well plates overnight at $37^{\circ} \mathrm{C}$. After transfection and culture for 14 days at $37^{\circ} \mathrm{C}$, cell colonies were formed in the dish and were fixed using $4 \%$ paraformaldehyde at room temperature for $15 \mathrm{~min}$ and stained using $0.5 \%$ crystal violet solution at room temperature for $10 \mathrm{~min}$ to manually count the numbers of colonies containing $>50$ cells. A cell group containing $>50$ cells was identified as a colony.

Wound-healing assay. Wound-healing assays were performed to detect the migratory ability of SW620 cells. The transfected SW620 cells were seeded in 6-well plates, allowed to reach $\sim 80 \%$ confluence and cultivated in serum-free medium for $24 \mathrm{~h}$. The cell monolayers were scratched vertically with a 200- $\mu 1$ RNase-free pipette tip. The width of the wound was captured at the indicated time points $(0$ and $24 \mathrm{~h})$ using an inverted fluorescence microscope (Olympus Corporation) (magnification, $\mathrm{x} 100$ ) to measure the percentage of the area covered by the migrated cells relative to the control.

Transwell assay. Cell invasive ability was assessed using Transwell assays. A Transwell chamber was precoated with 

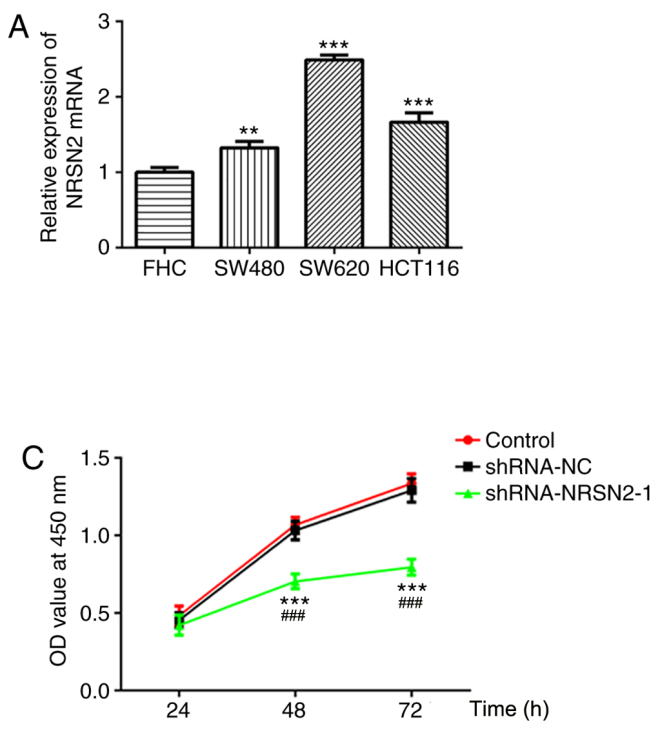
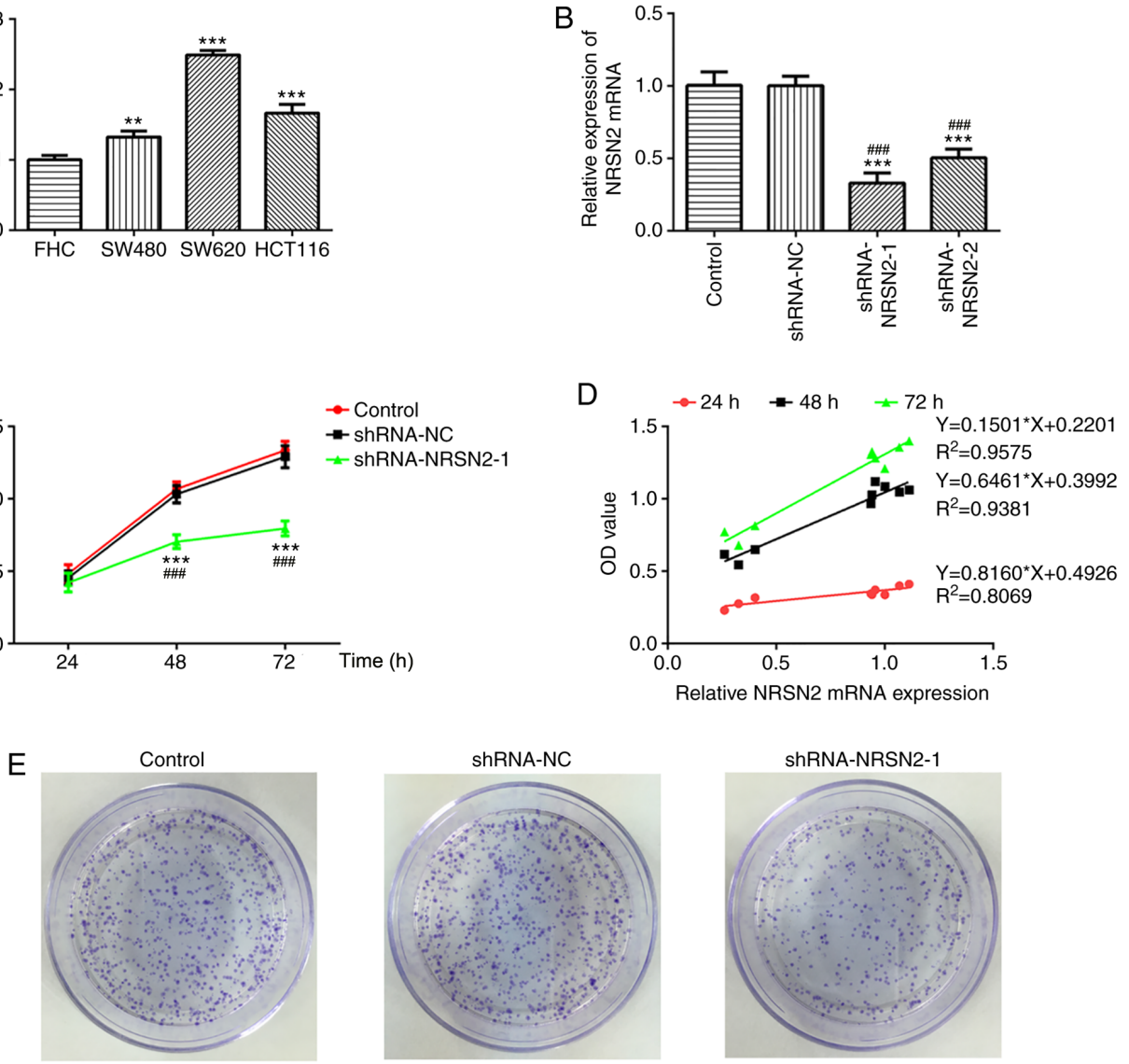

$\mathrm{F}$

Control

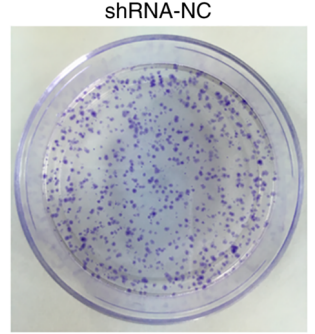

ShRNA-NC

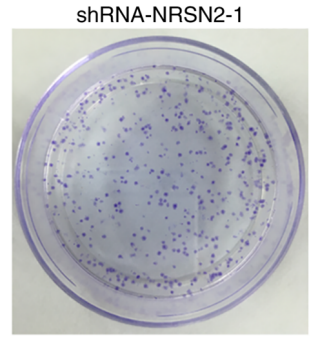

ShRNA-NRSN2-1
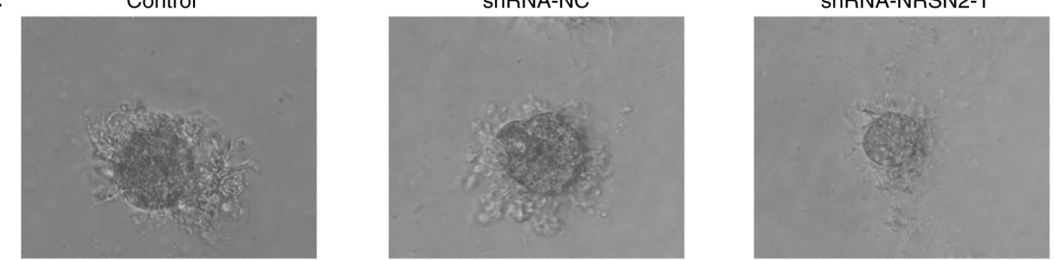

Figure 1. NRSN2 is highly expressed in CRC cells and NRSN2-knockdown inhibits the proliferation of SW620 cells. (A) mRNA expression of NRSN2 in CRC cells was evaluated via RT-qPCR.(B) mRNA expression of NRSN2 was evaluated via RT-qPCR to determine the transfection efficiency of shRNA-NRSN2. (C) Cell Counting Kit-8 assay was used to detect the viability of SW620 cells transfected with shRNA-NRSN2. (D) Correlation analysis was used to analyze the correlation between NRSN2 expression and cell viability in SW620 cells. (E) Colony formation assay was performed to determine the proliferative ability of SW620 cells transfected with shRNA-NRSN2 and (F) colony formation ability from a single cell captured with an inverted fluorescence microscope (magnification, $\mathrm{x} 400$ ). Data are presented as the mean \pm SEM from three independent experiments. ${ }^{* *} \mathrm{P}<0.01$ and ${ }^{* * *} \mathrm{P}<0.001$ vs. FHC or control; ${ }^{* \# \#} \mathrm{P}<0.001 \mathrm{vs}$. shRNA-NC. RT-qPCR, reverse transcription-quantitative PCR; shRNA, short hairpin RNA; NRSN2, neurensin-2; OD, optical density; NC, negative control.

Matrigel ${ }^{\circledR}$ (BD Biosciences) at $37^{\circ} \mathrm{C}$ for $30 \mathrm{~min}$. After transfection, SW620 cells $\left(1 \times 10^{5}\right.$ cells/ml in DMEM containing $1 \%$ bovine serum albumin) were seeded into the upper chamber and cultured at $37^{\circ} \mathrm{C}$ for $24 \mathrm{~h}$. DMEM containing $20 \%$ FBS was added into the lower chamber as a chemoattractant. Subsequently, the non-invasive cells were removed using a cotton-tipped swab and the cells in the lower chamber were fixed with $4 \%$ formaldehyde solution at room temperature for $15 \mathrm{~min}$ and stained with $0.1 \%$ crystal violet at $25^{\circ} \mathrm{C}$ for $20 \mathrm{~min}$. The stained cells were observed and counted manually under an inverted fluorescence microscope (Olympus Corporation) with three random fields at a magnification of x100.

Statistical analysis. Data are presented as the mean \pm SEM. Statistical analysis was performed using GraphPad Prism 5.0 (GraphPad Software, Inc.) using a two-sided unpaired Student's t-test for comparison between two independent groups or one-way ANOVA followed by Tukey's post hoc test to analyze differences among multiple groups. A Pearson's correlation analysis was used to analyze the relationship between NRSN2 expression level and cell growth in SW620 cells. $\mathrm{P}<0.05$ was considered to indicate a statistically significant difference.

\section{Results}

NRSN2 is highly expressed in CRC cells. To investigate the role of NRSN2, RT-qPCR and western blotting were performed to detect the expression levels of NRSN2 in CRC cells. The human CRC SW480, SW620 and HCT116 cell lines, and the human normal colon epithelial FHC cell line were used. RT-qPCR results demonstrated that NRSN2 expression was significantly increased in CRC cells, especially in SW620 cells, compared with FHC cells (Fig. 1A). Therefore, SW620 cells were selected for subsequent experiments. The results 

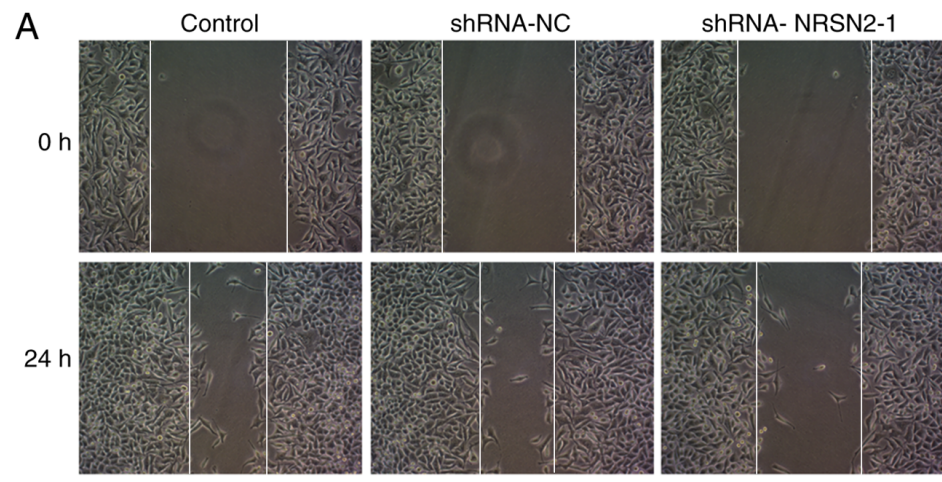

C

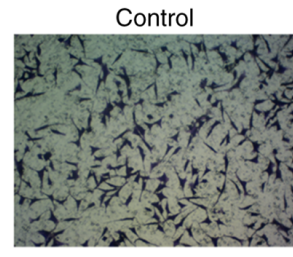

ShRNA-NC
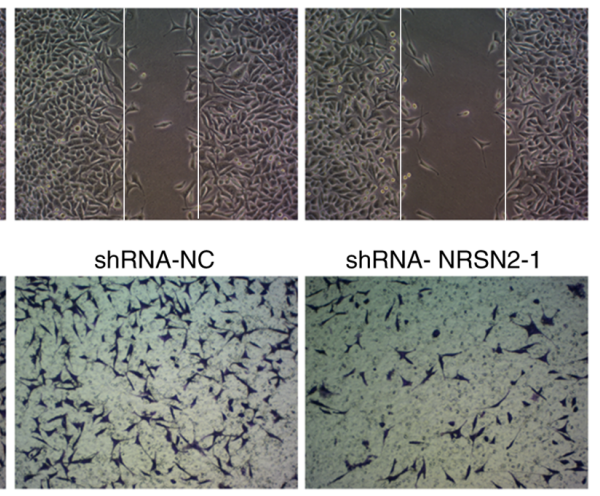

E
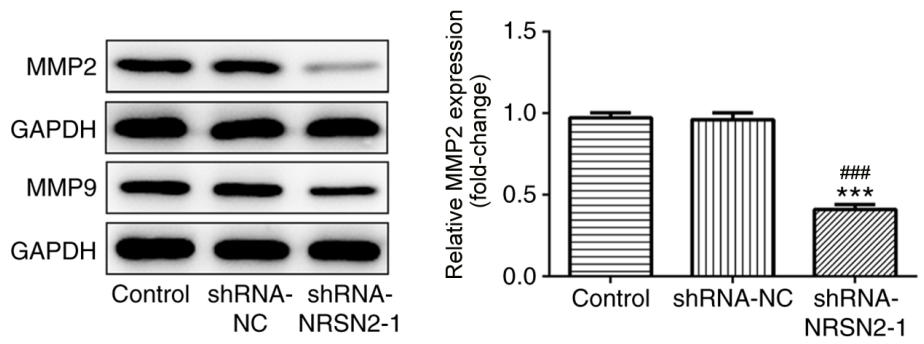
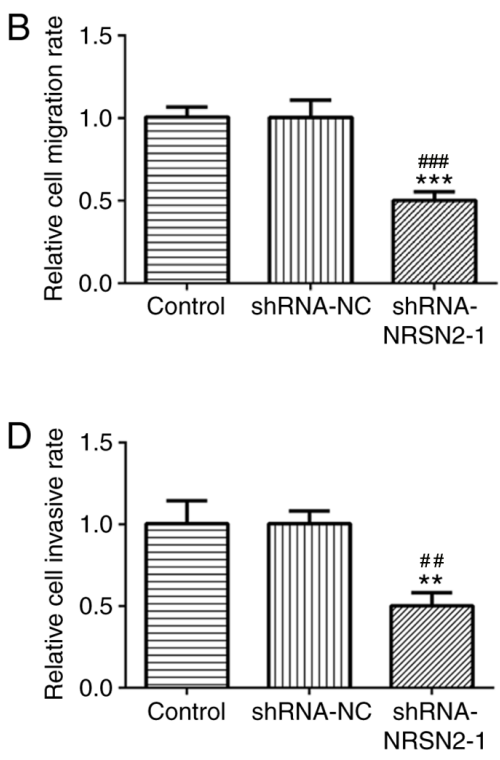

Figure 2. Effect of NRSN2-knockdown on the migration and invasion of SW620 cells. (A) Wound-healing assay was performed to (B) analyze the migratory ability of SW620 cells transfected with shRNA-NRSN2 (magnification, x100). (C) Transwell assay was used to (D) analyze the invasive ability of SW620 cells transfected with shRNA-NRSN2 at (magnification, x100). (E) MMP2 and MMP9 protein expression levels were analyzed via western blotting. Data are presented as the mean \pm SEM from three independent experiments. ${ }^{* *} \mathrm{P}<0.01,{ }^{* * *} \mathrm{P}<0.001$ vs. control; ${ }^{\# \#} \mathrm{P}<0.01,{ }^{\# \# \#} \mathrm{P}<0.001$ vs. shRNA-NC. shRNA, short hairpin RNA; NRSN2, neurensin-2; NC, negative control.

suggested that dysregulated NRSN2 expression may be closely associated with CRC pathogenesis.

Knockdown of NRSN2 inhibits proliferation, invasion and migration of CRC cells. To study the effect of NRSN2 on proliferation, invasion and migration of SW620 cells, shRNA-NRSN2 was constructed to knock down NRSN2 expression. According to RT-qPCR analysis, both shRNA-NRSN2-1 and shRNA-NRSN2-2 resulted in a significant decrease in NRSN2 expression, compared with control and shRNA-NC groups (Fig. 1B). Since shRNA-NRSN2-1 appeared to decrease NRSN2 expression to a greater extent compared with shRNA-NRSN2-2, shRNA-NRSN2-1 was selected for further experiments.

CCK-8 and colony formation assays were performed to determine the viability and proliferative ability of CRC cells. The correlation analysis was applied to analyze the relationship between NRSN2 expression level and cell growth in SW620 cells (Fig. 1D). Transfection with shRNA-NRSN2-1 significantly decreased the viability (Fig. 1C) and proliferative ability (Fig. 1E) of SW620 cells at 48 and $72 \mathrm{~h}$ compared with the control and shRNA-NC groups, as well as colony formation ability from a single cell (Fig. 1F). Wound-healing and Transwell assays were used to analyze the migratory and invasive abilities of SW620 cells, respectively. It was revealed that
SW620 cells had a decreased migratory capacity after transfection with shRNA-NRSN2-1 (Fig. 2A and B). Furthermore, the invasive ability of SW620 cells was significantly suppressed by transfection with shRNA-NRSN2-1 (Fig. 2C and D).

MMP2 and MMP9 expression levels are associated with tumor invasion and metastasis of malignant tumors (19). As measured via western blot analysis, shRNA-NRSN2-1 transfection led to a significant decrease in MMP2 and MMP9 expression (Fig. 2E). Overall, these findings indicated that knockdown of NRSN2 inhibited the proliferation, invasion and migration of SW620 cells.

Overexpression of NRSN2 promotes proliferation, invasion and migration of CRC cells. To evaluate the effect of NRSN2 on the proliferation, invasion and migration of SW620 cells, an NRSN2 plasmid was constructed to induce the overexpression of NRSN2. According to RT-qPCR and western blot analysis results, the NRSN2 plasmid significantly upregulated NRSN2 expression, indicating that the NRSN2 plasmid was successfully constructed (Fig. 3A and B).

Subsequently, CCK-8 and colony formation assays were performed to analyze the viability (Fig. 3C) and proliferative ability (Fig. 3D and E) of SW620 cells, which were significantly promoted by overexpression of NRSN2 at 48 and $72 \mathrm{~h}$. In addition, wound-healing and Transwell assay results suggested that 
A

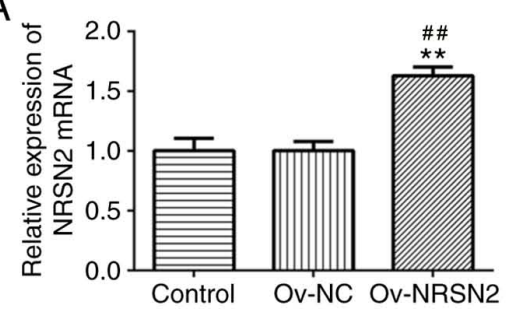

B

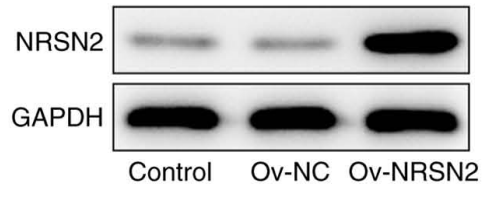

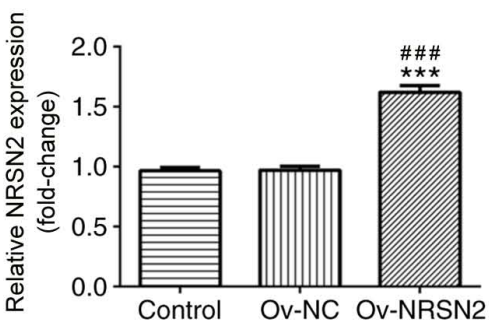

Ov-NC

$\mathrm{D}$

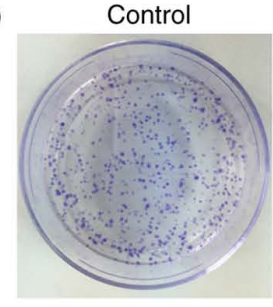

E

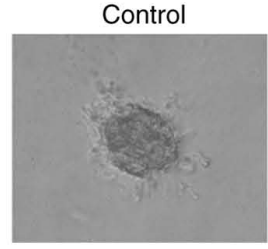

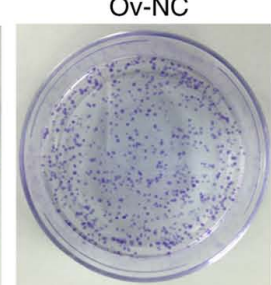

Ov-NC

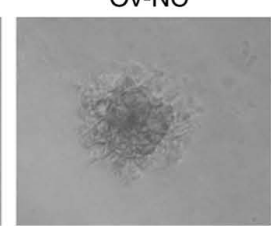

Ov-NRSN2-1

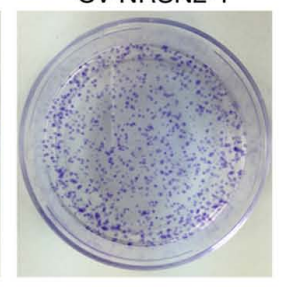

Ov-NRSN2-1

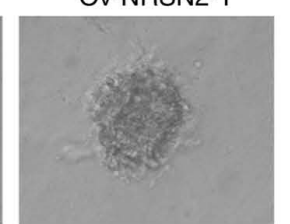

Figure 3. Effect of NRSN2 overexpression on the proliferation of SW620 cells. (A) Reverse transcription-quantitative PCR and (B) western blotting were performed to evaluate the mRNA and protein expression levels of NRSN2 to determine transfection efficiency of Ov-NRSN2, respectively. (C) Cell Counting Kit-8 assay was used to detect the viability of SW620 cells transfected with Ov-NRSN2. (D) Colony formation assay was employed to determine proliferation ability of SW620 cells transfected with Ov-NRSN2 and (E) colony formation ability from a single cell captured with an inverted fluorescence microscope

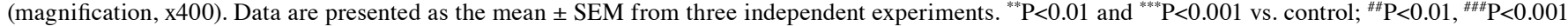
vs. Ov-NC. Ov, overexpression; NRSN2, neurensin-2; NC, negative control; OD, optical density.

overexpression of NRSN2 significantly increased the migratory (Fig. 4A and B) and invasive (Fig. 4C and D) ability of SW620 cells, respectively. The expression levels of MMP2 and MMP9 were evaluated using western blotting, revealing that they were significantly upregulated in SW620 cells transfected with Ov-NRSN2 (Fig. 4E). Therefore, the data suggested that NRSN2 overexpression promoted the proliferation, invasion and migration of SW620 cells.

SOX12 may be involved in the suppressive role of NRSN2 in $C R C$. To investigate the mechanism underlying the effect of NRSN2 promotion of the proliferation, invasion and migration of SW620 cells, the target proteins of NRSN2 were predicted using the STRING database (https://string-db. org/cgi/network), revealing that SOX12 may be recruited by NRSN2 (Fig. 5A). SOX12 expression is increased in CRC and promotes the proliferation, invasion and migration of CRC cells. Hence, SOX12 was chosen for investigation in the present study. Additionally, the physical association between NRSN2 and SOX12 was confirmed via IP in SW620 cells (Fig. 5B). The protein expression levels of SOX12 were determined after transfection with shRNA-NRSN2-1 and Ov-NRSN2 plasmid. As detected via western blot analysis, the results demonstrated that NRSN2-knockdown significantly decreased SOX12 expression, while NRSN2 overexpression significantly upregulated SOX12 expression, compared to control group. Overall, these results indicated that NRSN2 exerted a promoting effect on CRC tumorigenesis by recruiting SOX12.

\section{Discussion}

CRC has been identified as the fifth leading cause of cancer-associated mortality, with increasing incidence and mortality rates (20). For instance, it is estimated that there will be $>1.1$ million mortalities and 2.2 million new cases globally by 2030 due to CRC (21).

Previous studies have reported that various biomarkers can predict cancer progression and are associated with prognosis in patients with cancer $(13,22)$. In the present study, NRSN2 was observed to be associated with CRC tumorigenesis, and the current findings provided evidence to demonstrate that NRSN2 may serve a pivotal role in CRC progression. In addition, it is well-established that excessive proliferation of medial colorectal epithelial cells is a critical risk factor for CRC pathogenesis (23). According to the ATCC, the human colon cancer SW620 cell line isolated from the tissue of a Caucasian male was highly tumorigenic in nude mice (24), and has been employed in numerous genetic, biochemical and pharmacological studies $(25,26)$. Therefore, SW620 cells were used in the current study to determine the biological functions of NRSN2 in the development of CRC.

NRSN2, a novel gene that may act as a tumor promoter, encodes a small neuronal membrane protein (16). Tang et al (15) reported that NRSN2 expression is upregulated in ovarian cancer and serves an important role in tumor formation, anchorage-independent colony formation, cell invasion and chemoresistance. Additionally, Selga et al (27) demonstrated that NRSN2 is highly expressed in a 

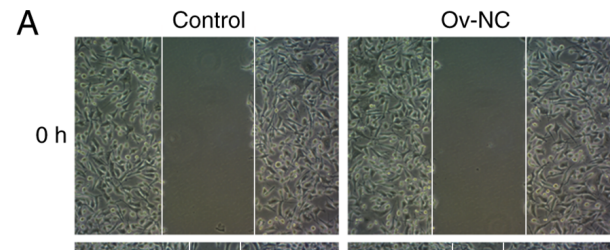

Ov-NRSN2-1
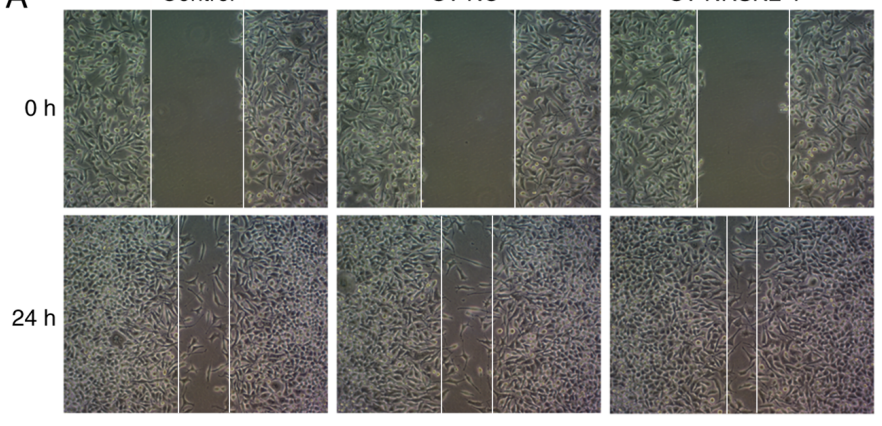

C

Control

Ov-NC

Ov-NRSN2-1
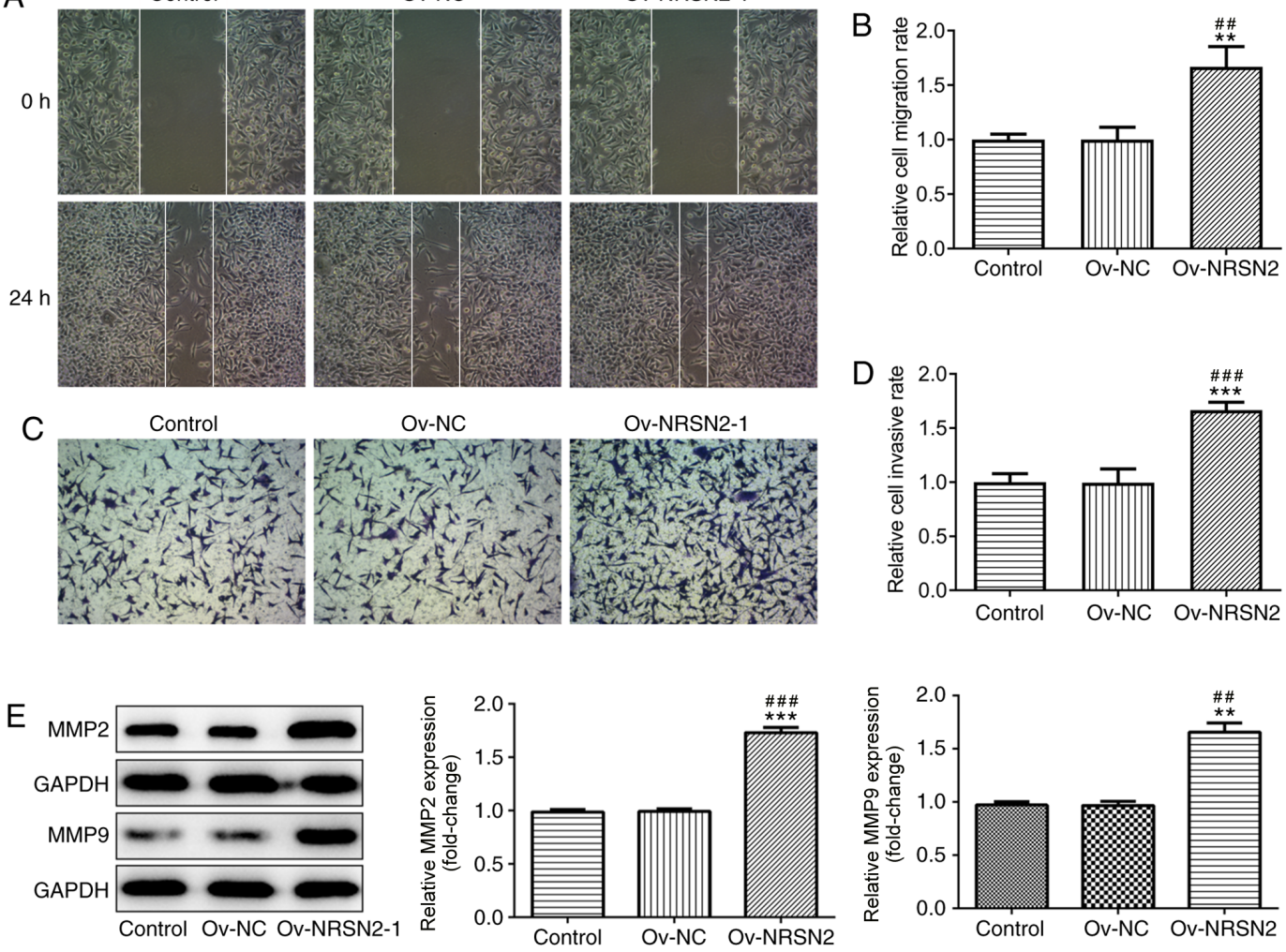

Figure 4. Effect of NRSN2 overexpression on the migration and invasion of SW620 cells. (A) Wound-healing assay was performed to (B) analyze the migratory ability of SW620 cells transfected with Ov-NRSN2 (magnification, x100). (C) Transwell assay was employed to (D) analyze the invasive ability of SW620 cells transfected with Ov-NRSN2 (magnification, x100). (E) MMP2 and MMP protein expression levels were analyzed via western blotting. Data are presented as the mean \pm SEM from three independent experiments. ${ }^{* *} \mathrm{P}<0.01$ and ${ }^{* * * *} \mathrm{P}<0.001$ vs. control; ${ }^{\# \#} \mathrm{P}<0.01,{ }^{\# \# \#} \mathrm{P}<0.001$ vs. Ov-NC. Ov, overexpression; NRSN2, neurensin-2; NC, negative control.
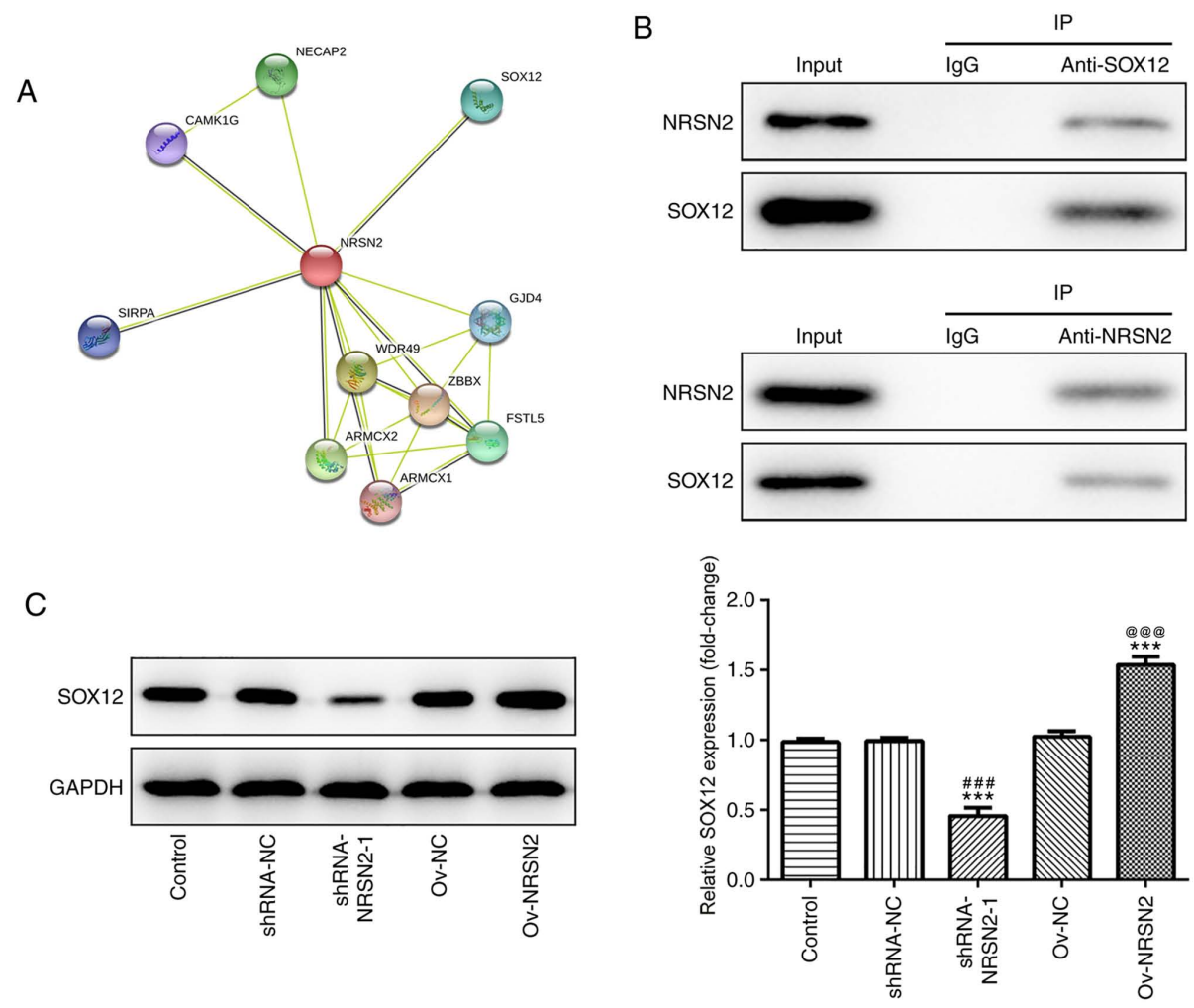

Figure 5. Association between NRSN2 and SOX12 in SW620 cells. (A) Interaction between NRSN2 and SOX12 was predicted using the STRING database. (B) IP was performed to confirm the association between NRSN2 and SOX12. (C) SOX12 protein expression was analyzed via western blotting. Data are presented as the mean \pm SEM from three independent experiments. ${ }^{* * *} \mathrm{P}<0.001$ vs control; ${ }^{\# \# / t} \mathrm{P}<0.001$ vs. shRNA-NC. ${ }^{\circledR @ @ ~} \mathrm{P}<0.001$ vs. Ov-NC. IP, immunoprecipitation; Ov, overexpression; NRSN2, neurensin-2; NC, negative control; shRNA, short hairpin RNA. 
methotrexate-resistant colon cancer cell line by searching the Gene Expression Omnibus database. Considering that NRSN2 exerts crucial roles in cancer progression, the present study analyzed NRSN2 expression in human CRC cells, revealing that NRSN2 was highly expressed in these cells, especially in SW620 cells. Subsequently, shRNA-NRSN2-1 was used for NRSN2 gene silencing and the Ov-NRSN2 plasmid was used for NRSN2 overexpression. NRSN2-knockdown suppressed the proliferation, invasion and migration of SW620 cells, while NRSN2 overexpression promoted these processes in SW620 cells, consistent with the aforementioned studies. Both loss- and gain-of-function assays indicated that NRSN2 promoted CRC cell proliferation and colony formation to facilitate cancer progression.

As predicted using the STRING database, SOX12 was a potential target protein of NRSN2. Furthermore, IP results demonstrated the interaction between NRSN2 and SOX12 in SW620 cells, and western blot analysis further confirmed an association between NRSN2 and SOX12. Chen et al (28) reported that the circular RNA hsa_circ_001895 sponges microRNA-296-5p to promote clear cell renal cell carcinoma progression by regulating SOX12. In addition, Du et al (29) demonstrated that SOX12 may represent a novel prognostic biomarker and regulator of gastric cancer metastasis via upregulating MMP7 and insulin-like growth factor 1. According to a previous study, SOX12 may serve as a prognostic biomarker of CRC, and promotes CRC cell proliferation and metastasis via regulating asparagine synthesis (30). In the present study, western blot analysis demonstrated that NRSN2-knockdown decreased SOX12 expression, while NRSN2 overexpression upregulated SOX12 expression, indicating that NRSN2 may exert accelerative effects on CRC progression by recruiting SOX12. Overall, the present findings suggested that NRSN2 may be a potential biomarker and may provide a novel therapeutic strategy for the treatment of CRC.

In conclusion, the present study demonstrated that NRSN2 promoted the proliferation, invasion and migration of CRC cells. SW620 cells were used to investigate the role of NRSN2 in CRC and to identify its mechanism, and the results suggested that NRSN2 exerted accelerative effects on CRC progression by recruiting SOX12. Therefore, NRSN2 may be used as a potential biomarker and promising therapeutic target for CRC. However, the interaction between NRSN2 and SOX12 has not been fully elucidated, and future experiments, such as invitro binding or co-localization assays, should be performed to understand the role of SOX12 underlying the effect of NRSN2.

\section{Acknowledgements}

Not applicable.

\section{Funding}

No funding was received.

\section{Availability of data and materials}

The datasets used and/or analyzed during the current study are available from the corresponding author on reasonable request.

\section{Authors' contributions}

GW was responsible for the writing of the manuscript, performed the experiments, analyzed and interpreted the data. KY reviewed the manuscript and designed the experiments. Both authors read and approved the final manuscript.

\section{Ethics approval and consent to participate}

Not applicable.

\section{Patient consent for publication}

Not applicable.

\section{Competing interests}

The authors declare that they have no competing interests.

\section{References}

1. González-Llorente L, Santacatterina F, García-Aguilar A, Nuevo-Tapioles C, González-García S, Tirpakova Z, Toribio ML and Cuezva JM: Overexpression of Mitochondrial IF1 Prevents Metastatic Disease of Colorectal Cancer by Enhancing Anoikis and Tumor Infiltration of NK Cells. Cancers (Basel) 12: 12, 2019.

2. Khelwatty SA, Essapen S, Bagwan I, Green M, Seddon AM and Modjtahedi $\mathrm{H}$ : Co-expression and prognostic significance of putative CSC markers CD44, CD133, wild-type EGFR and EGFRvIII in metastatic colorectal cancer. Oncotarget 10: 1704-1715, 2019.

3. Siegel R, Ma J, Zou Z and Jemal A: Cancer statistics, 2014 CA Cancer J Clin 64: 9-29, 2014.

4. Bray F, Ferlay J, Soerjomataram I, Siegel RL, Torre LA and Jemal A: Global cancer statistics 2018: GLOBOCAN estimates of incidence and mortality worldwide for 36 cancers in 185 countries. CA Cancer J Clin 68: 394-424, 2018.

5. Goldstein DA, Zeichner SB, Bartnik CM, Neustadter E and Flowers CR: Metastatic Colorectal Cancer: A Systematic Review of the Value of Current Therapies. Clin Colorectal Cancer 15: $1-6,2016$

6. Xu X, Zhang C, Xia Y and Yu J: Over expression of METRN predicts poor clinical prognosis in colorectal cancer. Mol Genet Genomic Med 8: e1102, 2020.

7. Dekker E, Tanis PJ, Vleugels JLA, Kasi PM and Wallace MB: Colorectal cancer. Lancet 394: 1467-1480, 2019.

8. Issa IA and Noureddine M: Colorectal cancer screening: An updated review of the available options. World J Gastroenterol 23: 5086-5096, 2017.

9. Allemani C, Matsuda T, Di Carlo V, Harewood R, Matz M, Nikšić M, Bonaventure A, Valkov M, Johnson CJ, Estève J, et al; CONCORD Working Group: Global surveillance of trends in cancer survival 2000-14 (CONCORD-3): Analysis of individual records for 37513025 patients diagnosed with one of 18 cancers from 322 population-based registries in 71 countries. Lancet 391: 1023-1075, 2018.

10. Pessaux P, Chenard MP, Bachellier P and Jaeck D: Consequences of chemotherapy on resection of colorectal liver metastases. J Visc Surg 147: e193-e201, 2010.

11. Siegel R, Desantis C and Jemal A: Colorectal cancer statistics, 2014. CA Cancer J Clin 64: 104-117, 2014.

12. Lima PMA, Torres LC, Martins MR, da Matta MC, Lima JTO, de Mello MJG, da Silva LM, Cintra EB Jr, Lira CCR, da Fonte EJA, et al: Soluble levels of sCD40L and s4-1BB are associated with a poor prognosis in elderly patients with colorectal cancer. J Surg Oncol 121: 901-905, 2020.

13. Leiphrakpam PD, Lazenby AJ, Chowdhury S, Smith LM, Mathiesen M, Brattain MG, Wang J, Black JD and Are C: Prognostic and therapeutic implications of NHERF1 expression and regulation in colorectal cancer. J Surg Oncol 121: 547-560, 2020. 
14. Keremu A, Maimaiti X, Aimaiti A, Yushan M, Alike Y, Yilihamu Y and Yusufu A: NRSN2 promotes osteosarcoma cell proliferation and growth through PI3K/Akt/MTOR and Wnt//-catenin signaling. Am J Cancer Res 7: 565-573, 2017.

15. Tang W, Ren A, Xiao H, Sun H and Li B: Highly expressed NRSN2 is related to malignant phenotype in ovarian cancer. Biomed Pharmacother 85: 248-255, 2017.

16. Zhang XY, Kuang JL, Yan CS, Tu XY, Zhao JH, Cheng XS and Ye XQ: NRSN2 promotes non-small cell lung cancer cell growth through PI3K/Akt/mTOR pathway. Int J Clin Exp Pathol 8 2574-2581, 2015.

17. Wang X, Han L, Zhang $J$ and Xia Q: Down-Regulated NRSN2 Promotes Cell Proliferation and Survival Through PI3K/Akt/mTOR Pathway in Hepatocellular Carcinoma. Dig Dis Sci 60: 3011-3018, 2015.

18. Livak KJ and Schmittgen TD: Analysis of relative gene expression data using real-time quantitative PCR and the 2(-Delta Delta C(T)) Method. Methods 25: 402-408, 2001.

19. Björklund M and Koivunen E: Gelatinase-mediated migration and invasion of cancer cells. Biochim Biophys Acta 1755: 37-69, 2005.

20. Li W, Xu Y, Wang X, Cao G, Bu W, Wang X, Fang Z, Xu Y, Dong $\mathrm{M}$ and Tao Q: circCCT3 Modulates Vascular Endothelial Growth Factor A and Wnt Signaling to Enhance Colorectal Cancer Metastasis Through Sponging miR-613. DNA Cell Biol 39: 118-125, 2020.

21. Zhu G, Lin C, Cheng Z, Wang Q, Hoffman RM, Singh SR, Huang Y, Zheng W, Yang S and Ye J: TRAF6-Mediated Inflammatory Cytokines Secretion in LPS-induced Colorectal Cancer Cells Is Regulated by miR-140. Cancer Genomics Proteomics 17: 23-33, 2020.

22. Wang XJ, Yu Q, Chi P, Lin HM, Lu XR, Huang Y, Xu ZB, Huang SH, Sun YW and Ye DX: Identification of gene biomarkers to predict responses to neoadjuvant chemoradiotherapy in patients with rectal cancer and pathways enrichment analysis. Zhonghua Wei Chang Wai Ke Za Zhi 22: 1183-1187, 2019 (In Chinese)
23. Dziki Ł, Puła A, Stawiski K, Mudza B, Włodarczyk M and Dziki $\approx A$ : Patients' Awareness Of The Prevention And Treatment Of Colorectal Cancer. Pol Przegl Chir 87: 459-463, 2015.

24. Huerta S, Harris DM, Jazirehi A, Bonavida B, Elashoff D, Livingston EH and Heber D: Gene expression profile of metastatic colon cancer cells resistant to cisplatin-induced apoptosis. Int J Oncol 22: 663-670, 2003.

25. 26. Melcher R, Steinlein C, Feichtinger W, Müller CR, Menzel T, Lührs H, Scheppach W and Schmid M: Spectral karyotyping of the human colon cancer cell lines SW480 and SW620. Cytogenet Cell Genet 88: 145-152, 2000

26. Broznić D, Ratkaj I, Malenica Staver M, Kraljević Pavelić S, Žurga P, Bubalo D and Gobin I: Evaluation of the Antioxidant Capacity, Antimicrobial and Antiproliferative Potential of Fir (Abies alba Mill.) Honeydew Honey Collected from Gorski kotar (Croatia). Food Technol Biotechnol 56: 533-545, 2018.

27. Selga E, Noé V and Ciudad CJ: Transcriptional regulation of aldo-keto reductase 1C1 in HT29 human colon cancer cells resistant to methotrexate: Role in the cell cycle and apoptosis. Biochem Pharmacol 75: 414-426, 2008.

28. Chen Z, Xiao K, Chen S, Huang Z, Ye Y and Chen T: CircRNA hsa_circ_001895 serves as a sponge of miR-296-5p to promote cell carcinoma progression via regulating SOX12. Cancer Sci 111: 713-726, 2020.

29. Du F, Feng W, Chen S, Wu S, Cao T, Yuan T, Tian D, Nie Y, Wu K, Fan D, et al: Sex determining region Y-box 12 (SOX12) promotes gastric cancer metastasis by upregulating MMP7 and IGF1. Cancer Lett 452: 103-118, 2019.

30. Du F, Chen J, Liu H, Cai Y, Cao T, Han W, Yi X, Qian M, Tian D, Nie Y, et al: SOX12 promotes colorectal cancer cell proliferation and metastasis by regulating asparagine synthesis. Cell Death Dis 10: 239, 2019.

This work is licensed under a Creative Commons Attribution-NonCommercial-NoDerivatives 4.0 International (CC BY-NC-ND 4.0) License. 